\title{
Multidisciplinary Optimization of a Turboelectric Tiltwing Urban Air Mobility Aircraft
}

Eric S. Hendricks*, Robert D. Falck ${ }^{\dagger}$, Justin S. Gray ${ }^{\ddagger}$, Eliot D. Aretskin-Hariton ${ }^{\S}$, Daniel J. Ingraham ${ }^{\mathbb{I}}$, Jeffryes W. Chapman", Sydney L. Schnulo**, Jeffrey C. Chin ${ }^{\dagger \dagger}$, John P. Jasa ${ }^{\dagger \neq}$, and Jennifer D. Bergeson ${ }^{\S \S}$ NASA Glenn Research Center, Cleveland, $\mathrm{OH}, 44135$

Urban air taxis, also known as urban air mobility (UAM) vehicles, are anticipated to be an area of significant market growth in the near future. These vehicles are typically vertical take-off and landing (VTOL) designs which are capable of carrying 1 to 30 passengers in an intra-urban environment with flights of less than 50 nautical miles. Development of UAM vehicles and their integration into the airspace will be enabled by advancements in a number of areas including electrified propulsion systems, structures, acoustics, automation, and controls. However, the strong multidisciplinary interactions for these unique vehicles presents a significant new design challenge. This work describes the development of a multidisciplinary analysis and optimization environment which can be used to support the conceptual design of these UAM vehicles, using efficient gradient based optimization with analytic derivatives. The tools included in this multidisciplinary analysis model the aircraft trajectory, vehicle aerodynamics, structures, and electrified propulsion system. The multidisciplinary environment created in this research is unique in that all the physics tools are tightly integrated together, with the trajectory model directly calling the aerodynamics, structures, and propulsion models. This multidisciplinary analysis environment is then demonstrated in the design optimization of a turboelectric tiltwing UAM vehicle concept.

\section{Nomenclature}
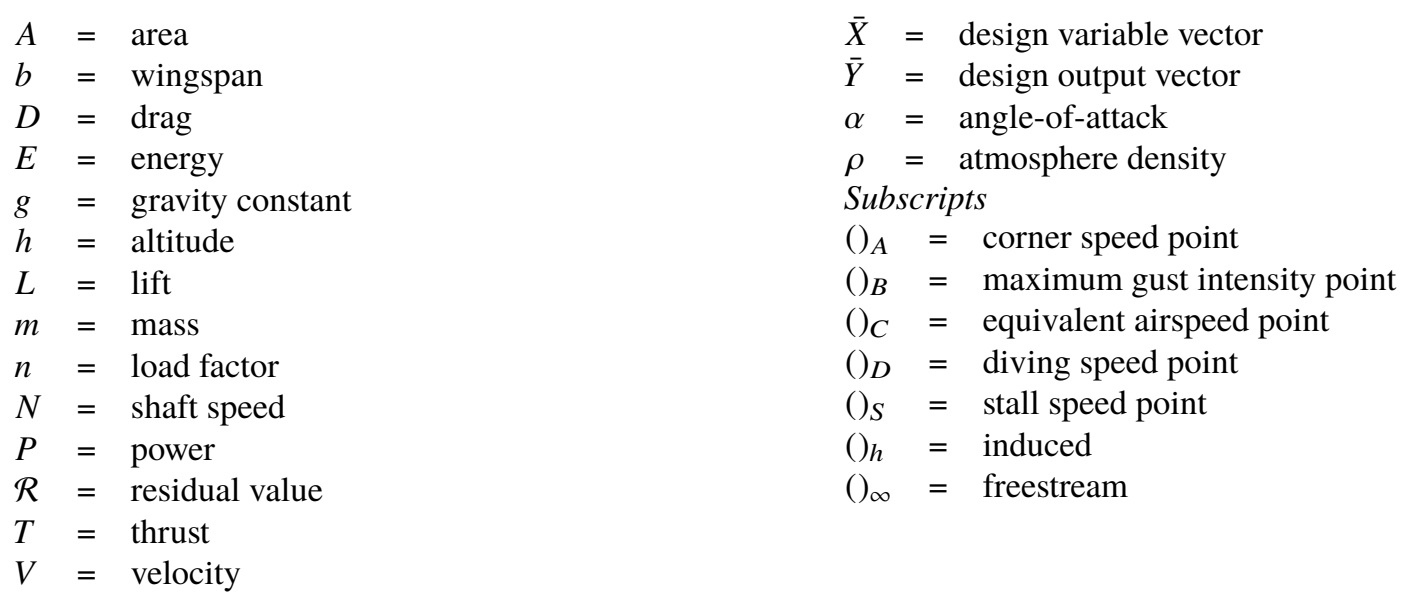

\footnotetext{
*Aerospace Engineer, Propulsion Systems Analysis Branch, 21000 Brookpark Rd.

$\dagger$ Aerospace Engineer, Mission Analysis and Architecture Branch, 21000 Brookpark Rd, AIAA Member

$¥$ Aerospace Engineer, Propulsion Systems Analysis Branch, 21000 Brookpark Rd, AIAA Member.

${ }^{\S}$ Aerospace Engineer, Intelligent Control and Autonomy Branch, 21000 Brookpark Rd.

II Aerospace Engineer, Acoustics Branch, 21000 Brookpark Rd.

"Aerospace Engineer, Propulsion Systems Analysis Branch, 21000 Brookpark Rd.

**Aerospace Engineer, Propulsion Systems Analysis Branch, 21000 Brookpark Rd.

${ }^{\dagger}$ Aerospace Engineer, Propulsion Systems Analysis Branch, 21000 Brookpark Rd.

$\$$ Aerospace Engineer Intern, Propulsion Systems Analysis Branch, 21000 Brookpark Rd and Ph.D. Candidate, University of Michigan.

$\S$ Aerospace Engineer Intern, Propulsion Systems Analysis Branch, 21000 Brookpark Rd and Undergraduate Student, Purdue University.
} 


\section{Introduction}

$\mathrm{F}^{\mathrm{OR}}$ more than half a century, humans have envisioned a future where every person would have a personal air vehicle

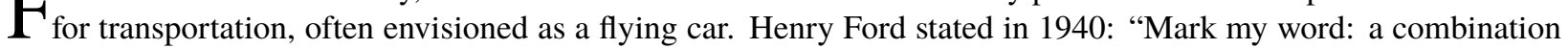
airplane and motorcar is coming. You may smile, but it will come."'[1] While this future has yet to come to pass, recent developments in aviation and technology have made this reality closer than ever. The vision now is for an urban air taxi service (also commonly referred to as urban air mobility or UAM) capable of shuttling passengers around metropolitan areas thereby escaping the road congestion below.[2] This vision has sparked the development of a number of UAM concept vehicles within industry and at NASA. These vehicles are typically vertical take-off and landing (VTOL) designs which which are capable of carrying 1 to 15 passengers in an intra-urban environment on flights of less than 50 nautical miles.

Within NASA, four UAM concept vehicles were initially developed by the Revolutionary Vertical Lift Technology (RVLT) Program to focus and guide research activities. [3, 4] These vehicles, shown in Fig. 1. differ significantly in their design, size, payload, range, propulsion system and operation. The concept on the left is a quadrotor vehicle designed to carry a single passenger over a short $50 \mathrm{~nm}$ range. Because of this short range and small size, it is anticipated that the propulsion system for this vehicle would be all-electric. The two vehicle on the right are larger with the ability to carry six passengers on multiple flights before needing refueling. The side-by-side rotor concept (2nd from right) is envisioned to have a hybrid propulsion system with some power produced by energy stored in batteries with the rest coming from turboshaft engines while the Lift+Cruise concept (right) is a candidate for either electric or turboelectric propulsion systems. Lastly, the largest concept is a tiltwing design (2nd from left) capable of carrying 15 passengers on eight $50 \mathrm{~nm}$ flight segments. The propulsion system for this concept is expected to be a turboelectric design where a single turboshaft engine generates electricity which is transmitted to four electric motor driven rotors/propellers. It is this last concept, the turboelectric tiltwing, which will serve as the demonstration example in this paper.

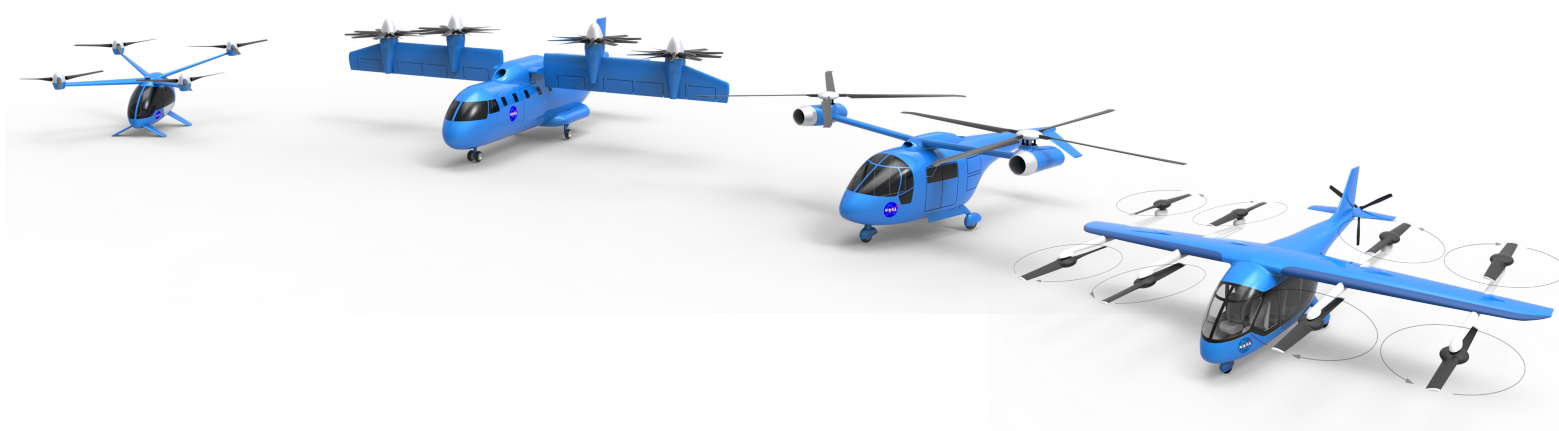

Fig. 1 NASA Urban Air Mobility Concept Vehicles.

The initial studies to develop these UAM concept vehicle designs applied a set of existing rotorcraft design and analysis tools in a traditional design process. Overall, the the objectives of these studies were to sufficiently refine the design such that crucial technologies and research needs could be identified. As a result, the generated concept designs revealed several areas of further research which are required to evolve and mature the designs were identified. These areas included improving the modeling and assumptions for the propulsion system, vehicle weights, aerodynamics and acoustics. [3] Furthermore, it was identified that the traditional design process needs to be modified to perform an integrated multidisciplinary environment to optimize these concepts to further improve the overall design

This paper presents a new design process that uses a tightly coupled multidisciplinary analysis environment for conceptual design of UAM vehicles. This new design process combines tightly integrated disciplinary analysis for aircraft trajectory, propulsion, electrical, structures and aerodynamics with the application of gradient-based optimization with analytic derivatives to better analyze vehicle concepts. The use of analytic derivatives provides significant computational savings when gradient-based optimization is employed as they reduce the overall computational cost by orders of magnitude [5] and enable parametric studies to be performed by looking at sets of optimized aircraft designs. Although the use of analytic derivatives does pose some potentially significant implementation challenges, the more commonly used alternative of finite-difference derivative approximation has been shown to be costly and numerically unstable. [6, 7] The OpenMDAO framework [8] was chosen as the computational platform for implementing the tightly-coupled 
multidisciplinary optimization, in order to take advantage of its support for modular model construction and automatic analytic derivative computation which greatly reduces the associated implementation challenges.

The objective of the research presented in this paper is to develop and demonstrate the methods required for a tightly-coupled multidisciplinary optimization. Available tools for each discipline were used where possible with the understanding that those tools might not accurately capture the physics of the complicated UAM concept designs. These tools limit the ability to assess the quality of the designs produced at this time, but enable a demonstration of the tightly-coupled process with plans for the disciplinary tools to be improved in future iterations of this work.

This paper is organized as follows: Section III first presents the traditional design process and tools used in the initial design of the UAM concept vehicles. Following this background, the development of the new multidisciplinary design, analysis and optimization (MDAO) environment and the supporting disciplinary tools are summarized in Section IV The features of the multidisciplinary analysis environment are then demonstrated by applying it to the optimization of the turboelectric tiltwing UAM vehicle described above in Section V. Following this optimization demonstration, conclusions and future work are discussed in Section VI.

\section{Traditional Design and Analysis Environment}

The initial UAM concepts described in the Introduction were developed by the RVLT research team using a fairly traditional design process. This process, described by the Extended Design Structure Matrix (XDSM) [9] in Fig. 2] in a multidisciplinary design and optimization (MDO) context would typically be called a iterative sequential design optimization. In the figure, three individual design disciplines are shown as an example, though addition additional disciplinary analyses can be added as required.

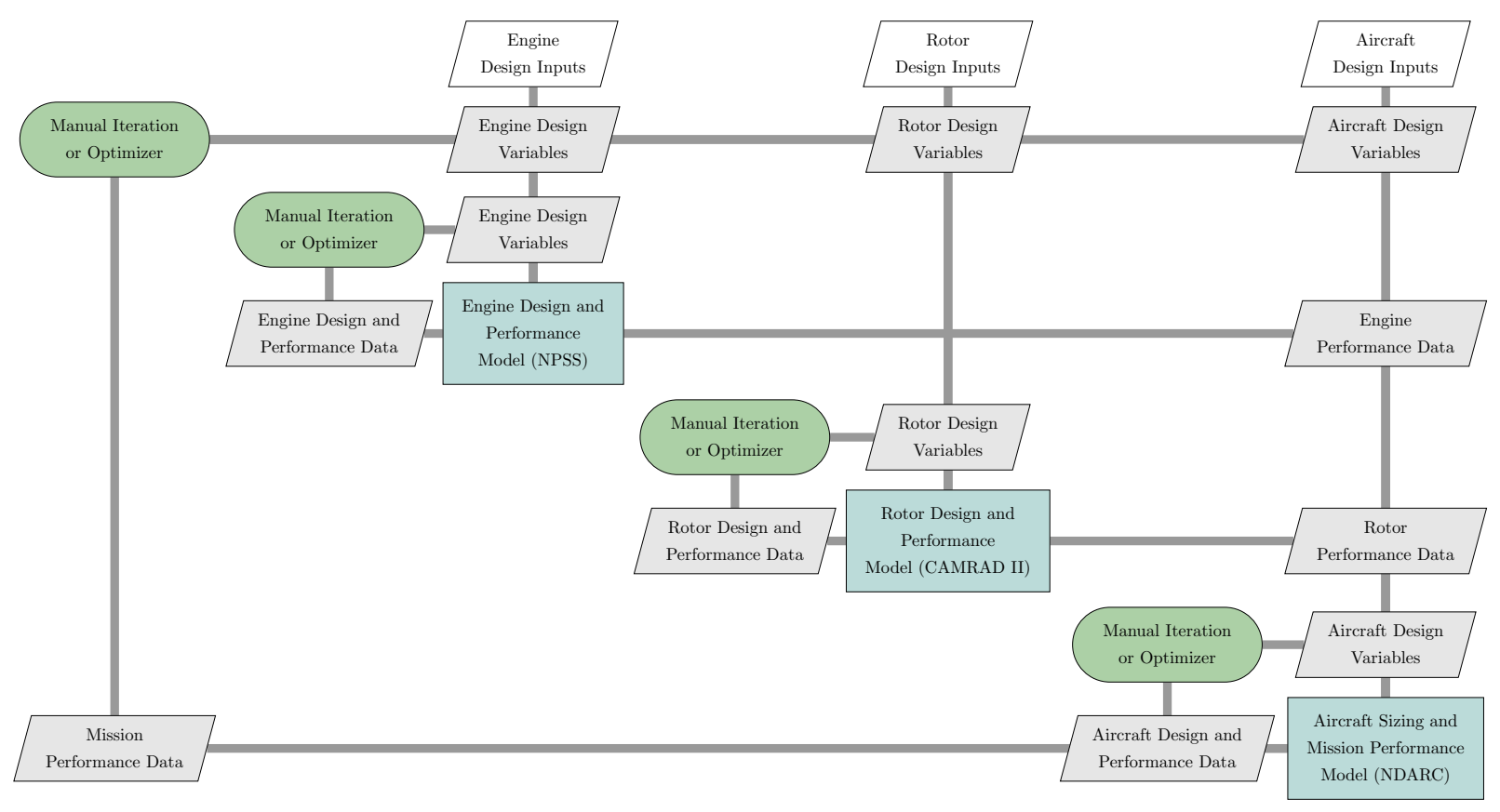

Fig. 2 Traditional Conceptual Design Process.

The first discipline shown in this example process is for the turboshaft engine and commonly uses the Numerical Propulsion System Simulation (NPSS) code.[10] This model both develops both the engine design and evaluates its performance over a wide range of operating conditions. In the traditional process, a numerical optimizer or a series of manual iterations may be used to develop an engine suitable for the given aircraft and mission. The result of this analysis is the generation of engine performance data which is required by later steps in the design process. This data is often used to generate a data table that can be interpolated or reduced order model to determine performance characteristics for any feasible flight condition in a trajectory analysis.

Next, a similar process is used to design the rotor(s) and determine the performance characteristics. The reference 
configurations developed by the RVLT project, used the Comprehensive Analytical Model of Rotorcraft Aerodynamics and Dynamics (CAMRAD II) code for this portion of the design process.[11-13] Like the engine analysis, design of the rotor(s) may include manual iterations or application of an optimizer to generate a desirable design. The resulting rotor performance data is also passed onto the trajectory analysis in the form of tabular data or a reduced order model.

The last disciplinary analysis model included in the generic process shown in Fig. 2 2 is for the overall aircraft design and trajectory performance evaluation. This analysis develops and sizes the full vehicle then computes its performance over the mission(s) of interest. The RVLT project used NASA Design and Analysis of Rotorcraft (NDARC) code for this step in their development of the reference configurations.[14] Like the previous disciplines, manual iterations or optimization can be used around the aircraft design and mission performance model to improve the design. This vehicle design iteration/optimization typically occurs, however, without significant modification of the previously developed engine and rotor designs.

Therefore, if major limitations in the vehicle performance are uncovered an outer iteration or optimization loop might be required. This outer loop, represented by the green oval in the top left of the figure, could be performed manually or automatically. If manually completed, discipline experts would leverage their tools in each step to re-design their sub-systems to the newly updated requirements. If automatically executed, a multidisciplinary framework can be used to automate the data passing between analysis tools and an optimizer can be wrapped around the overall model. The RVLT concept vehicles were developed with a combination of manual iteration and automated data passing using NASA's OpenMDAO framework [8], where discipline experts preselected key design aspects for each system ahead of time and then integrated models were exercised over a narrowed design space to refine concepts.

While this overall process and the constituent tools have historically worked well for developing conceptual rotorcraft designs, the traditional process has several weaknesses in regards to developing designs for emerging UAM vehicle concepts. Since the existing process centers around the sequential application of disciplinary tools to the design of vehicle sub-systems, the only real mechanism for sub-system interactions to be accounted for is the experience and intuition of the separate disciplinary experts. In the context of an multidisciplinary design optimization, this is referred to as loose coupling, which has been shown to produce suboptimal designs in other fields such as aerostructural wing design.[15] The sequential process also becomes unwieldy as additional disciplinary analyses - e.g. electrical power distribution, thermal management, and acoustics - become more important and need to be considered in the conceptual design process. The inclusion of thermal management models presents an additional challenge as it has been shown that transient thermal models integrated into the trajectory analysis are required to capture performance constraints. [16, 17] One final practical consideration is that the introduction of new tightly coupled disciplinary analyses into the design process significantly increases the complexity and computational cost of the model, while at the same time decreasing its numerical stability. The decrease in numerical stability comes from the increased likelihood that at least one code will fail and the difficulties that arise in computing gradient information across coupled models.

\section{Multidisciplinary Design, Analysis, and Optimization Environment}

As described in the Introduction, the UAM concept development to date has focused on identifying a set of vehicle designs and the discipline areas where further refinement is needed to improve the designs. Given the multidisciplinary design challenges presented by these new UAM vehicle concepts and the limitations of the existing design process, the objective of this research was to develop a multidisciplinary analysis and optimization environment capable of further exploring and refining these designs. Specifically, this work focused on enabling tight coupling of multiple disciplines (in comparison to the loose coupling of the traditional process) to directly capture interaction effects, while as the same time addressing the practical challenges that such coupling creates. The overall multidisciplinary design environment and the constituent disciplinary analysis tools are summarized in this section.

To develop this tightly-coupled multidisciplinary environment, NASA's OpenMDAO framework [8] was used as a computational platform for two primary reasons. First, it provided a low-level computing library upon which new disciplinary analyses with analytic derivatives needed for this research could be built. The disciplinary analysis built on top of the OpenMDAO library are described later in this section. Second, it provided a framework to integrate these various disciplinary analysis tools together to form the overall multidisciplinary environment and compute derivatives across the integrated model for gradient based optimization. These features made OpenMDAO a valuable platform for developing and implementing the desired multidisciplinary environment.

To describe the multidisciplinary design and optimization environment created in this research, several XDSM diagrams are presented here. These diagrams depict the overall model components, execution sequence, and more importantly the data which couples the various disciplines to form a tightly integrated analysis process. The first XDSM 


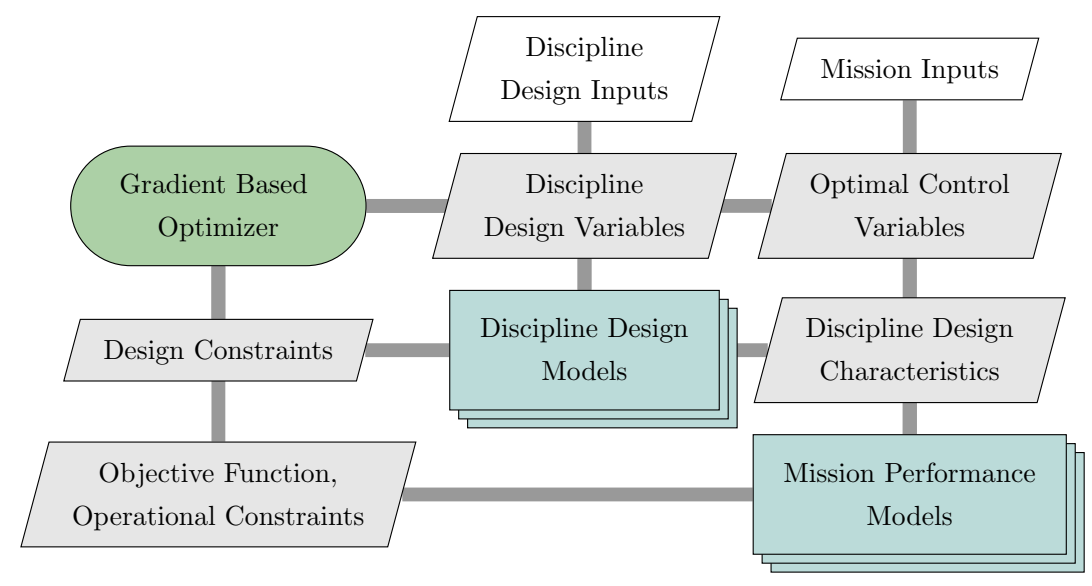

Fig. 3 Multidisciplinary Analysis Environment.

in Fig. 3 outlines the overall multidisciplinary design environment used in this work. This design environment is broadly decomposed into three major elements which are shown along the diagonal of the figure. The first element is a gradient based optimizer which drives the execution of the various models by altering design and control variables to achieve a target design objective subject to a set of constraints. The second major element is a set of discipline design models which size the aircraft components for a given set of performance requirements. Information about the design of these components is then passed to the last major element which evaluates the mission performance of the vehicle given the control values selected by the optimizer.

While at the top-level the overall multidisciplinary environment shown in Fig. 3 appears similar to the traditional design process, the developed environment differs significantly in terms of the disciplinary models and how they are coupled. In particular, the traditional process often evaluates the detailed disciplinary models only in the design element with reduced order models (in the form of an interpolated table or surrogate model) passed to the mission performance element. For example, the aircraft sizing and mission performance evaluation in NDARC used in the traditional process relies on input data tables of propeller and turbine engine performance in its computations. By comparison, in the present work there is a desire to move towards directly utilizing actual physical models throughout the analysis to better capture physical coupling that is important to overall performance. Therefore, the current work utilizes the detailed discipline models wherever possible within both the disciplinary design and mission performance elements. While there is a desire to use these detailed models, in some cases it can be valuable to implement only the reduced order model (based on results from a high-fidelity analysis) in both elements particularly when the computational cost of the detailed model is high and design freedom with the discipline is limited. This flexibility in modeling approach allows for the appropriate level of modeling for each discipline to be implemented for the vehicle design under consideration making the proposed design environment an adaptable framework for analyzing a variety of vehicle concepts.

The integration and coupling of disciplinary models throughout both the design and mission performance elements for the multidisciplinary environment is detailed more thoroughly in Figures 4 to 6 First, Fig. 4 depicts the disciplinary analyses completed as part of the design element. In this portion of the analysis, the propulsion system components including the propellers/rotors, electrical system and turboshaft engine are first evaluated. Coupling is achieved among these disciplines primarily by ensuring the power demands by the various components are consistent throughout the analysis. For the propulsion system portion of this assessment, the most demanding or constraining hover condition is used to size the various elements of the system. Following the execution of the propulsion system elements, the wing aerostructural design is completed and used to determine the overall aircraft mass. The wing aerostructural design considers multiple critical stress conditions in forward flight and was therefore evaluated at different operating conditions than the propulsion system portion of the disciplinary design calculations. Execution of the components in the disciplinary design phase requires an iterative process as the propulsion system and wing design require an initial estimate of overall aircraft mass to develop the designs. Therefore, a numerical solver is included in this portion of the design environment to generate a converged solution.

Overall, the design process depicted in Fig. 4 is very similar to the standard aircraft sizing analysis that can be found 


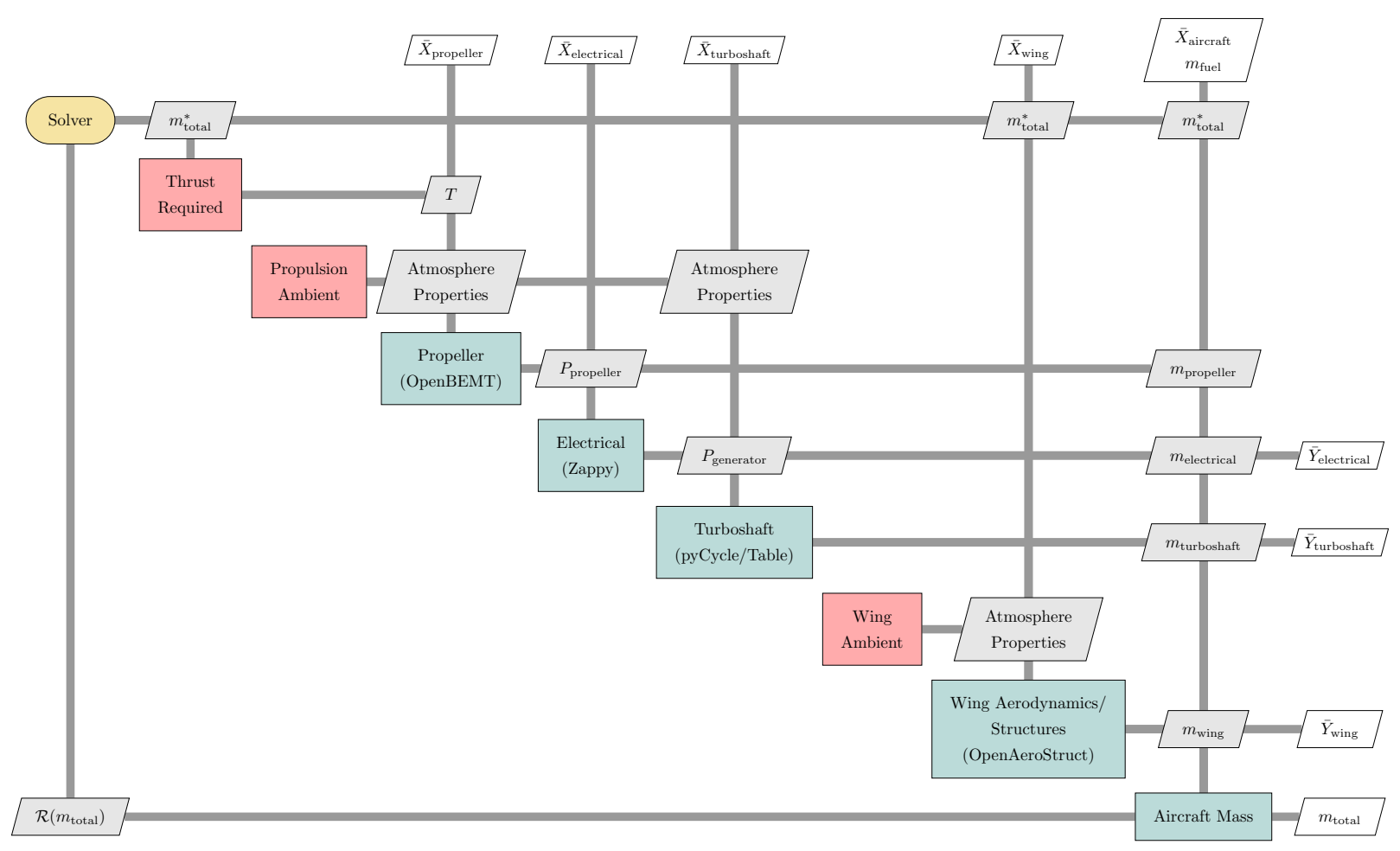

Fig. 4 Discipline Design Modeling.

in aircraft conceptual design textbooks. However, some models include more detailed analysis than would normally be seen in the textbook process such as the aerostructural analysis that uses a vortex-lattice aerodynamic method coupled to a beam based structural model to estimate the wing weight. Also, the propeller performance is modeled directly using a blade element momentum theory based approach rather than relying on a performance map. Note that it is not necessary, or even recommended, that every single discipline be modeled using a detailed analysis tool. For example, the turbine engine performance can be modeled directly using a 1-D thermodynamic model (pyCycle) or with a basic interpolation off a pre-computed data table. For this work, the pre-computed data-table approach was used for the cycle analysis as previous studies showed that there was not enough design freedom in the thermodynamic design to warrant tight coupling of this discipline with the rest of the system. [18]

For the mission performance modeling element of the overall process, two separate models were constructed for evaluating the performance of a tiltwing UAM concept. The first, shown in the XDSM in Fig. 5. contains the disciplinary models for computing the aircraft's performance characteristics in vertical flight (hover) while the second, shown in Fig. 6, computes the performance in forward flight.

For both of these mission performance modeling elements, the execution is completed using a transient modeling library called Dymos. [19] Dymos, which itself is built on top of OpenMDAO, implements a general ordinary differential equation (ODE) integration capability that enables use of several different time integration schemes. This implementation includes the calculation of analytic derivatives for the different integration schemes to better enable the construction and solution of the transient analysis. For this work, the Radau pseudospectral scheme was selected for its relatively low computational cost (compared to an explicit scheme such as RK4) and good computational stability. The use of Dymos enables a full mission trajectory analysis to be included, rather than relying on a fixed, pre-determined reference trajectory. While a fixed trajectory could be modeled, in theory this assumption may not produce the best mission performance available as the suboptimal trajectory can impact the design of the vehicle. In the specific case examined in this work, the results ultimately showed that a fixed trajectory would have been a reasonable assumption. However, this result is not known a-priori and therefore might not be general enough to handle a larger design space exploration.

While Dymos is used to drive the mission performance analyses for both vertical and forward flight, it requires a 


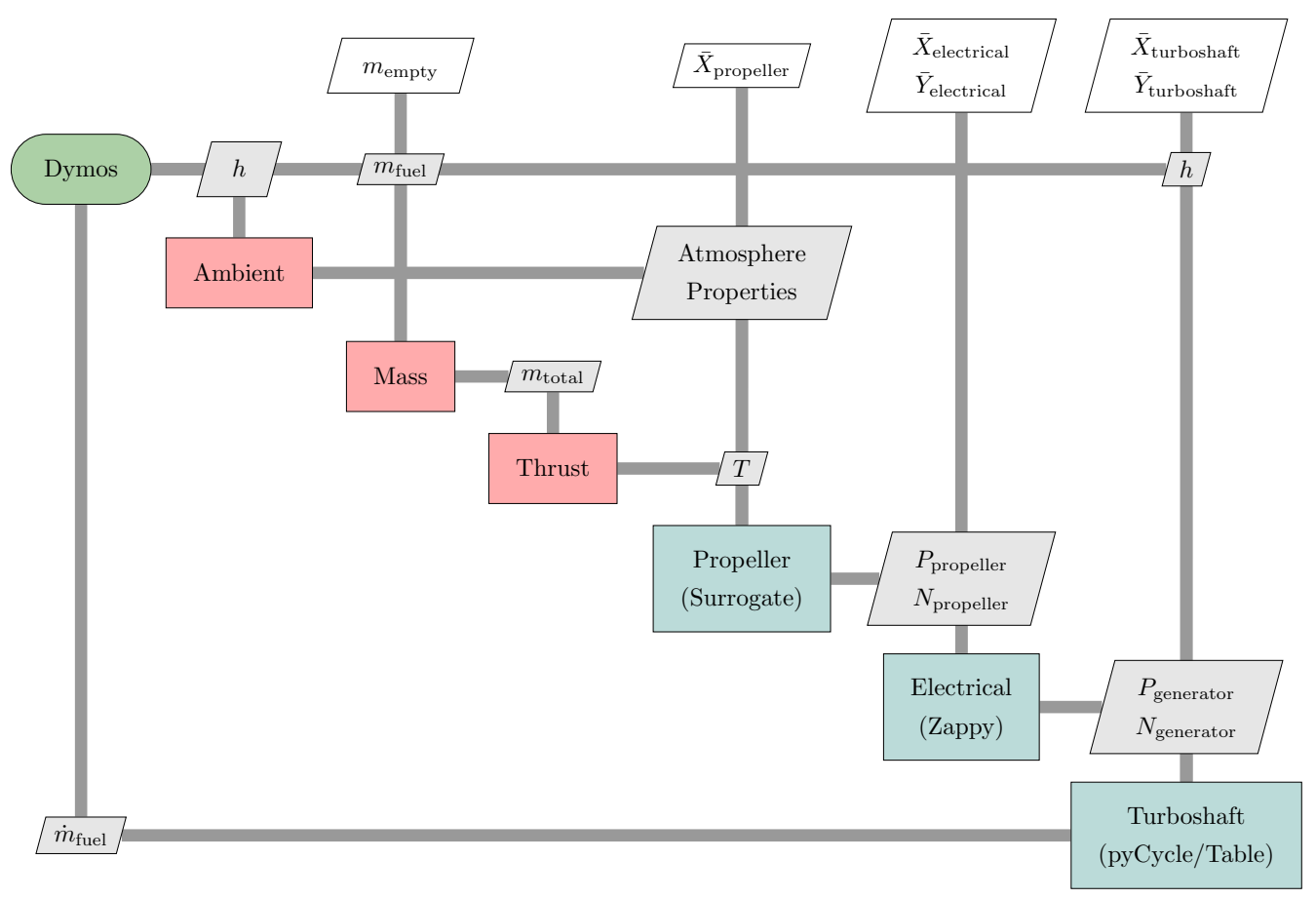

Fig. 5 Vertical Flight Performance Modeling.

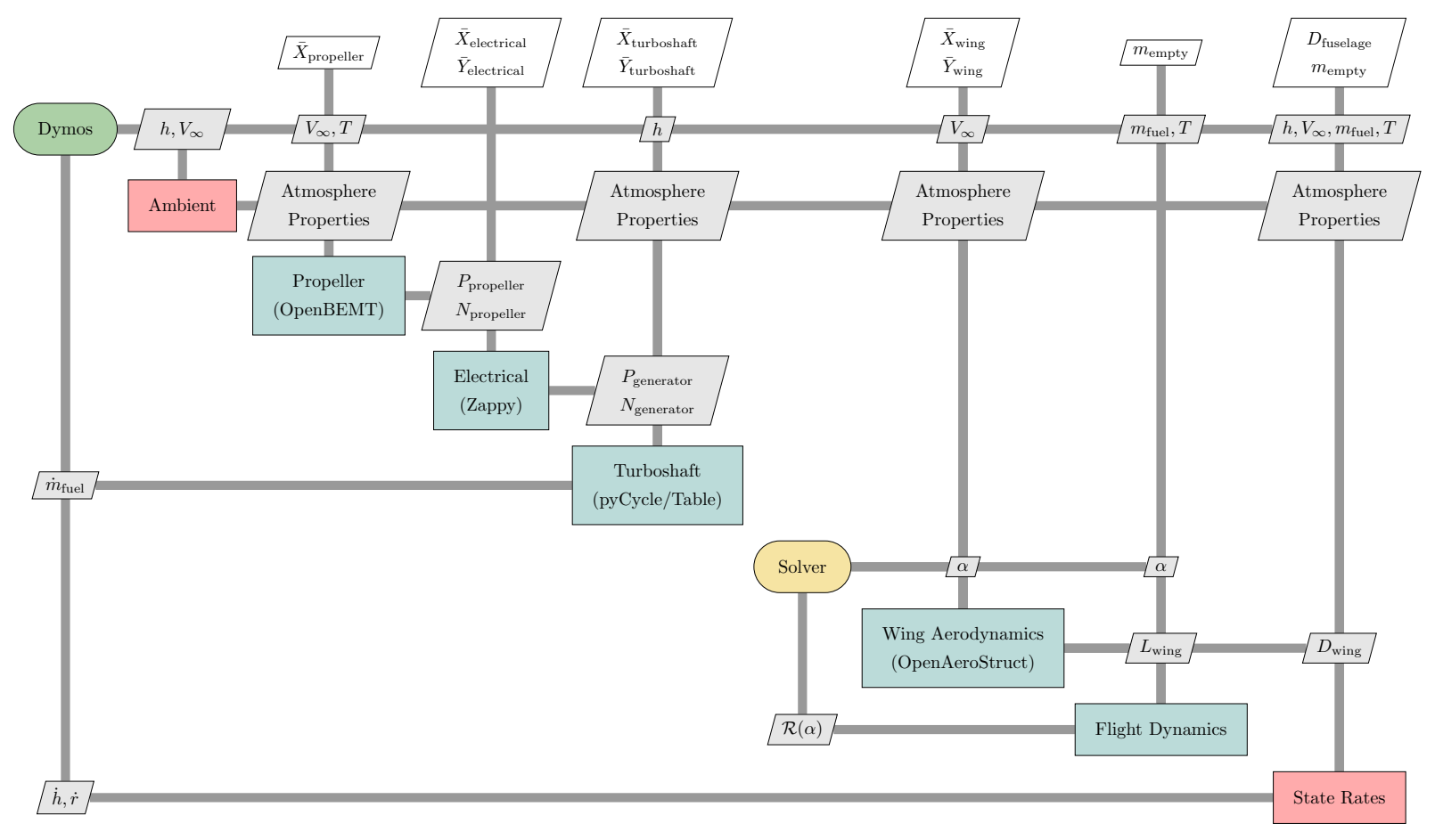

Fig. 6 Forward Flight Performance Modeling. 
specific ODE sub-model consisting of the disciplinary analyses to complete the actual aircraft trajectory analysis. In this portion of the analysis, each of the individual models are evaluated at a large number of time steps throughout the mission trajectory. As shown in Fig. 5 for vertical flight, the analysis starts by computing the weight and required thrust of the vehicle. This thrust value is then passed to the propeller analysis, which in this phase is in the form of a surrogate model, to determine the required propeller shaft power and ultimately the power that must be supplied by the gas turbine. The gas turbine cycle analysis computes a fuel flow rate that is then used by Dymos to ensure valid flight dynamics.

For forward flight a different set of disciplinary analyses is required as shown Fig. 6 As shown in this figure, the three propulsion system components are evaluated again at each time step to determine the required fuel flow given an input thrust demand. Following the propulsion system components, the wing aerodynamic performance is evaluated to support calculation of the flight dynamics. For forward flight, a solver balance is required around the wing and flight dynamics elements to vary the aircraft angle of attack to achieve the desired rate of climb. The data generated by the various disciplines at each flight point is then used to compute state rates which are the outputs of the ODE sub-model that Dymos numerically integrates.

Although the multidisciplinary design environment presented in these figures could be constructed from existing disciplinary tools, this research focused on applying disciplinary tools that each provide analytic derivatives. This approach is unique and is a significant difference as the accurate and rapid calculation of these derivatives within OpenMDAO is critical to generating the total derivatives across the entire design loop needed for gradient-based optimization. It must be emphasized that in this initial iteration of the research, the focus was primarily on establishing the multidisciplinary environment and the methodology for tightly coupling the individual disciplines. The discipline tools and the quality of their physical models were therefore not of primary concern, so it is recognized that there are significant limitations associated with ability of the tools selected to properly model the UAM concepts under consideration. The tools used were selected based on their computational cost and the availability of analytic derivatives with plans to improve the level of physics captured by the disciplinary tools in future iterations. The next sections will provide additional details about the individual disciplinary modeling tools that provide these derivatives which were applied as part of this research.

\section{A. Propulsion System Analysis}

The propulsion systems under consideration for NASA's UAM concept vehicles cover a range of different system configurations from all-electric to turbo-electric architectures. This variation makes choosing a single propulsion modeling tool set problematic. For the tiltwing vehicle examined in this work, three different analysis tools were used to model the full propulsion system: a blade element momentum theory analysis for rotor or propeller performance, a load flow based electrical system analysis, and 1D gas turbine thermodynamic cycle analysis. The overall propulsion system model is shown in Fig. 7 , where the gray boxes represent the four rotors/propellers, the red boxes show elements of the electrical system model, and the blue boxes show the components modeled in the thermodynamic cycle. This section describes the models built with those tools in more detail, but it is important to note that future work could use different propulsion analysis tools as appropriate.

\section{Rotor/Propeller Analysis}

The propeller/rotor aerodynamics are predicted in this research using blade element momentum theory (BEMT). Although BEMT codes rely on fairly simple physics, they commonly pose significant numerical challenges due to lack of stability of the nonlinear solvers used to converge them. The specific BEMT implementation used in this work is a modified formulation developed by Andrew Ning which was designed to have guaranteed convergence, specifically for optimization use cases. [20] This implementation, called OpenBEMT, has been previously used to predict performance for a design optimization of an aircraft based on NASA's X-57 electric distributed propulsion concept, [21] and it has also been used as part of a proof-of-concept coupled trajectory-acoustic optimization for the small single person RVLT UAM quad-rotor concept. [22] In this work, OpenBEMT is used to predict the performance of the tiltwing's four propellers with the simplifying assumption that each propeller was an isolated system and therefore the analysis did do not capture interactions between the individual rotors or between the wing and the rotors. These assumptions are significant limitations to the quality of the physical representation produced by this modeling tool, and it recognized that higher-fidelity physics models are necessary for this discipline to capture rotorcraft performance in future work. However, the exiting implementation of OpenBEMT provided a relatively fast tool that could output thrust and required shaft power, which get passed to the flight dynamics calculations and electrical system analysis respectively.

For the discipline design condition, the inflow velocity was assumed to be approximately $6.5 \mathrm{feet} / \mathrm{second}$ ( 2 


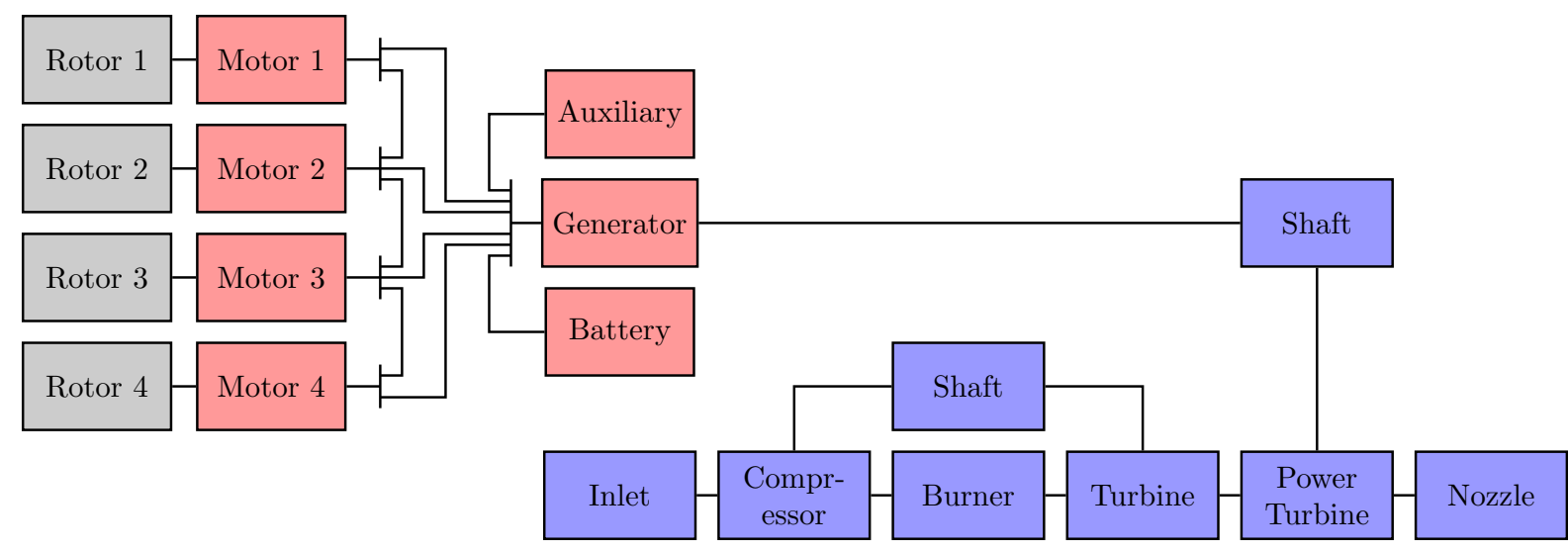

Fig. 7 Propulsion System Model Components.

meters/second), corresponding to a vertical takeoff condition. The blade inner diameter was also fixed at about 12 inches ( 30 centimeters), and the outer diameter was set so that each pair of propellers covered the entire wing span with a small overlap allowed. The design variables considered in the BEMT model were the chord and twist distributions of the propeller rotors, and for any given design the rotational rate was solved for to provide the required thrust for the vertical take off maneuver.

In the mission performance portion of the environment, OpenBEMT was run directly coupled to the trajectory analysis which was allowed to vary propeller pitch angle throughout the flight. The vehicle's forward velocity was used as the inflow velocity, without taking into account the vehicle's angle of attack as it was assumed that the angle of attack will be small throughout the trajectory. The propeller's rotational rate was again calculated implicitly by finding the value that gives the total thrust value demanded by the flight dynamics.

\section{Electrical System Analysis}

To model the electrical components of the propulsion system, a new power systems modeling library called Zappy was developed on top of the OpenMDAO framework. This tool is based on the calculations and methods used in hybrid AC-DC load flow or power flow analysis [23] with additional computational calculations added to capture motor, generator and battery performance.

In order to use this tool, the user must first define the electrical system layout including the major components and the cables connecting those components. Additionally, performance information for each of the components in the system must be specified. This information primarily includes performance maps for the motors and generators (efficiency as a function of shaft speed and torque) as a well as impedances in the cables. Furthermore, the electrical system takes as input the power demanded by each of the rotors, the power required to operate auxiliary systems, as well as the output voltage provided by the generator. With this information, the electrical system model can be executed to determine the power that must be produced by the generator as well as the power that is lost to heat in the various components throughout the electrical system. The analysis of the electrical system is nearly identical between the disciplinary design and mission analysis phases.

The electrical system model for the tiltwing includes the components shown in red in Figure 7 . The model includes four electric motors, which each includes an integrated inverter, that are connected to each of the four rotors. These motor components therefore require DC power which is supplied by an interconnected system of DC cables and electrical buses. The microgrid produced by this interconnected system was assumed to provided redundancy in the system in the event that failures occurred in one or more lines. At the other end of the microgrid, a generator and its integrated rectifier are used to supply DC power to the system. A battery is also included in the model to supply emergency backup power in the event of failure of the gas turbine engine. Given this operational assumption, the battery is not charged or discharged during the mission performance modeling. However, it was sized to provide two minutes of emergency power in the disciplinary design modeling with the battery weight included in the overall mass buildup. 


\section{Thermodynamic Cycle Analysis}

The gas turbine portion of the propulsion system was modeled using a new analysis tool for thermodynamic cycle analysis. The code, called pyCycle [24, 25], was selected for this study as it is also built on top of the OpenMDAO framework. pyCycle differs from other thermodynamic cycle analysis tools such as NPSS in that it provides analytic derivatives to better support gradient-based optimization of the gas turbine as part of larger multidisciplinary optimization studies.

Modeling a gas turbine engine with pyCycle first necessitates that the designer specify the major engine components (i.e. compressor, combustor, turbine, etc.) as well as how these components are connected (both via in terms of flow and shafts). For each of these components, the user must also provide information about the performance characteristics of the components. In the disciplinary design phase, this commonly includes information such as the design point component efficiencies, pressure ratios, and performance maps. In addition, during the design phase the maximum hover power required must be given as an input. With this information, the gas turbine engine is sized during the design phase with the outputs being the engine mass as well as the flow areas and map scalars which are passed to the model evaluated in the mission analysis phase. For the mission analysis phase, the same model and connections are evaluated as the design phase with some minor variations in the calculations. For this phase, the model again takes in the required shaft power needed by the generator and then determines the fuel flow rate subject to maximum temperature limits.

For the tiltwing turboelectric concept under consideration in this paper, the pyCycle model developed is represented by the blue portion of Figure 7 and is described in more detail in a paper by Chapman. [18] In summary, the flowpath for the turboshaft engine in this study includes an inlet, compressor, burner, turbine, power turbine and nozzle. Furthermore, the compressor and turbine are connected by a shaft with the power turbine connected to the generator by a separate shaft. While not included in the simplified graphic of Figure 7, the model also includes calculations to compute the required turbine cooling flows as well as the effects of small compressor blade heights on compressor efficiency. For computing the weight of the engine in this study, a simple correlation was developed from historical data for turboshaft engines based on the overall airflow through the engine, which was presented in Chapman work. Previous studies of this engine [18] showed that thermodynamic cycle performance and weight were relatively insensitive to major design parameters such as overall pressure ratio and combustor exit temperature. Therefore, given the complexity of the cycle analysis calculations, the detailed cycle model was pre-executed with a surrogate model of the performance characteristics used in the integrated analysis.

\section{B. Wing Aerodynamic and Structural Analysis}

To model the aerostructural performance of the wing, an open-source tool for preliminary sizing of aircraft wings called OpenAeroStruct [26] was selected. OpenAeroStruct uses a vortex-lattice method coupled with a 6-degree-offreedom-per-node finite element model to analyze the aerostructural properties of aircraft wings. These inexpensive physics-based methods enable rapid exploration of the wing design space which is valuable for conceptual level design studies.

Using OpenAeroStruct, a model was constructed to represent the wing of the tiltwing vehicle concept under consideration in this study. The initial overall geometry (wingspan and root chord) were obtained from Johnson et al. [3] with other aerostructural wing properties assumed based on similarly sized aircraft. The relevant values for these parameters which were used in this study are listed in Table 1. In addition to these parameters, a NACA 0015 symmetric airfoil was assumed for the wingbox cross-section, with this section occupying from $10 \%$ to $70 \%$ chord for the entire wing span. The wing was also assumed to be constructed from Aluminum 2024 for the lower skin and spars and Aluminum 7075 for the upper skin with a safety factor of 1.5 applied on the yield strength of the wingbox material. Finally, two point masses were included on each half-wing to account for the loading due to the engines and propellers with the magnitude of these masses coming from the upstream propulsion sizing analyses. A three-view and isometric view of the aerostructural wing model including the approximate locations of the engines are shown in Fig. 8 .

This wing model is used in both the discipline design and mission performance analyses. For discipline design, a 3-point aerostructural analysis was performed to ensure that the internal structure of the wing does not fail during limit loading. The limiting loading conditions for this analysis are produced from a $V-n$ diagram, which shows the aircraft limit load factor as a function of equivalent airspeed (EAS). [27] EAS is the airspeed at sea level in a standard atmosphere where the dynamic pressure is the same as the dynamic pressure at the true airspeed. [27] This $V$ - $n$ diagram is constructed using both the worst-case maneuver and gust loads to find the flight conditions where the wing structure is most likely to fail. The $V-n$ diagram produced for this wing and nominal flight conditions is shown in Fig. 9 In this figure, $V_{S}$ is the stall speed at normal level flight, $V_{A}$ is the corner speed, $V_{B}$ is the design speed for maximum gust 
Table 1 Aerostructural Wing Properties.

\begin{tabular}{lrl}
\hline Design parameter & Value & Units \\
\hline Wingspan & 52.50 & feet \\
Root chord & 4.56 & feet \\
Elastic modulus & 73.1 & $\mathrm{GPa}$ \\
Shear modulus & 27.5 & $\mathrm{GPa}$ \\
Yield strength (lower skin and spars) & 324 & $\mathrm{MPa}$ \\
Yield strength (upper skin) & 503 & $\mathrm{MPa}$ \\
Yield safety factor & 1.5 & - \\
Wingbox material density & 2810 & $\mathrm{~kg} / \mathrm{m}^{3}$ \\
\hline
\end{tabular}

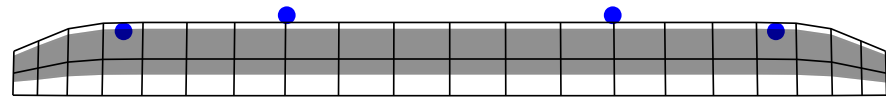

Fig. 8 Three-View and Isometric View of the Aerostructural Wing Model with the Engine Point Masses Shown in Blue.

intensity, $V_{C}$ is the design equivalent airspeed, $V_{D}$ is the design diving speed. Also included in this figure are the gust lines from Federal Aviation Regulation (FAR) 25, which provide airworthiness standards for transport airplanes. [28]

The $V-n$ diagram describes a range of different loading flight conditions that may need to be evaluated in the disciplinary design phase, with the most critical points determined by a number of assumptions. For this study, $V_{C}$ was assumed to be the max cruise speed specified by Johnson et al. [3] Also, a maneuver loading of $2.5 \mathrm{~g}$ and dive speed to cruise speed ratio of 1.25 were assumed. Finally, it was assumed that each of these these limit loading flight conditions were to be evaluated at an altitude of 5000 feet. Based on these assumptions and Fig. 9, the most critical loads were identified as being at $2.8 \mathrm{~g}$ and $-1 \mathrm{~g}$ due to gust loading and push-over conditions, respectively. Therefore, this study used $1 \mathrm{~g},-1 \mathrm{~g}$, and $2.8 \mathrm{~g}$ flight conditions in the 3-point aerostructural analysis completed in the discipline design.

For the mission performance analyses, the wing model was executed such that it only evaluated the aerodynamic performance without considering the wingbox structure. Evaluating only the aerodynamics in the mission performance phase is appropriate for two primary reasons. First, the aircraft is not expected to experience structurally limiting loads during the nominal mission being evaluated. Second, although there will undoubtedly be some wing deflection during climb and cruise segments of the flight, this deflection has a negligible effect on the aerodynamic performance. Therefore, the wing deflection obtained from the $1 \mathrm{~g}$ aerostructural analysis completed in the disciplinary design phase was applied to the wing mesh used in the aerodynamics only analysis completed in the mission performance phase. Given these reasons, removing the structural portion of the analysis was deemed acceptable and thereby enabled a reduction in computational cost during this phase. 


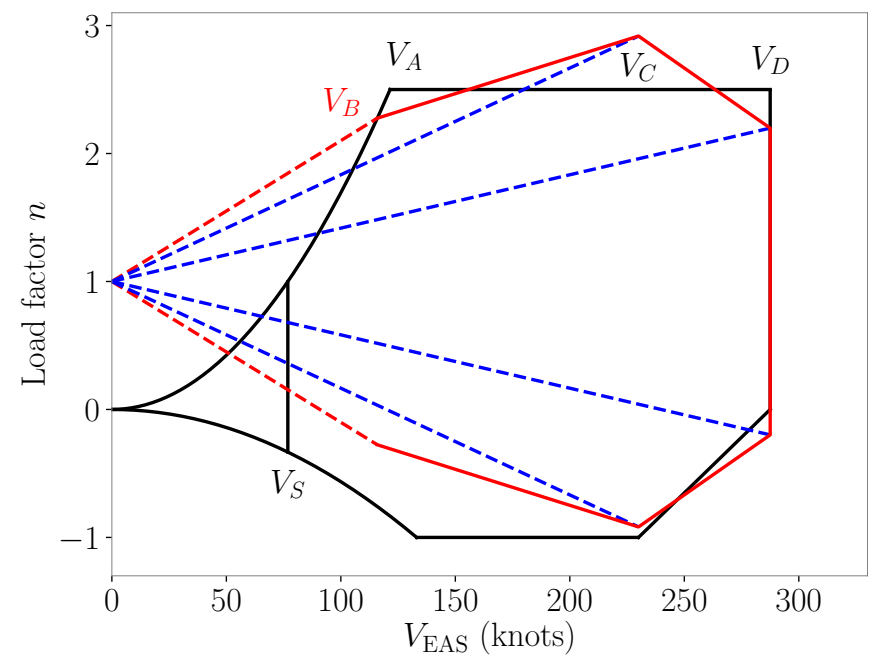

Fig. 9 V-n Diagram Used to Determine the Limiting Flight Conditions for the Wing Structure.

\section{Aircraft Mass Analysis}

In addition to the structural analysis completed for the wing, the disciplinary design phase includes additional calculations to estimate the overall mass of the aircraft. This estimation is broken down by major aircraft subsystem with empirical correlations used to approximate the weight for the subsystems.

For the aircraft structure, empirical correlations were used following the methods applied by NDARC, [14] which are often based on the U.S. Army Aeroflightdynamics Directorate (AFDD) preliminary design methods with the exception of the wing weight which came from OpenAeroStruct. First, the fuselage mass was based on the AFDD84 NDARC universal body weight model. In addition, the tail weight was computed from the AFDD82 NDARC model and included both the horizontal and vertical tail components. Fuel system weight includes tanks and supporting structures, and is based on a 15 aircraft regression. The landing gear and alighting structural masses were based on a regression of 28 aircraft.

For the propulsion system related elements, the mass estimating equations came from a more diverse set of correlations. The propeller weight was based on the NDARC AFDD82 model which used 35 aircraft to empirically determine hub and blade weights. Next, the drive system weight including gearbox, rotor break, and shaft was included and is based on the NDARC AFDD83 regression based on 30 aircraft. For the electric system, the motor, inverter, rectifier and generator weights are all estimated based on linear regressions of flightweight systems presently in development for aircraft. These regressions, given in Equations (1) and (2), output mass in kilograms for input power in kilowatts.

$$
\begin{aligned}
& m_{\text {motor }}=m_{\text {generator }}=0.18 P_{\text {required }} \\
& m_{\text {inverter }}=m_{\text {rectifier }}=0.0616 P_{\text {required }}+0.6165
\end{aligned}
$$

For the electric cabling, an empirical regression that computes mass in units of mass per unit length, as as function of maximum power through any one line, was used. The max cable power represented a worst-case failure where several cables are severed, with these calculations occurring as part of design calculations at max hover power. The cable length was computed based on wing-span, with an assumed 4.2 cable runs to each of the four motors, as given in Equation (3).

$$
m_{\text {cable }}=0.0028 b P_{\text {cable }}
$$

For the battery, the weight was estimated based on an assumed usable specific energy density of $400 \mathrm{Wh} / \mathrm{kg}$ with a capacity computed such that the battery could provide 2 minutes of emergency hover power. Finally, the turboshaft engine weight was approximated using a linear regression based on shaft power for existing turboshaft engines from Chapman. [18] 


\section{Flight Dynamics}

For the tiltwing aircraft under consideration in this study, the trajectory is modeled as two distinct forms of flight. The trajectory begins and ends with vertical ascent/descent phases which propagate the change in altitude and fuel mass subject to a commanded climb rate. Between these two vertical flight phases, the bulk of the trajectory is evaluated in a forward flight mode which is modeled using a steady-flight assumption. Between the vertical flight and forward flight portions of the trajectory, the aircraft must go through a brief transition phase. The transition phase is not modeled in the present work due to its more complex physics and short duration, however it may be included in future studies. The sections below describe the flight dynamics implemented in the vertical and forward flight phases of the trajectory.

\section{Vertical Flight}

In vertical flight, the trajectory of the aircraft is controlled by specifying the climb rate at any given time. In this analysis, the climb rate is assumed to be constant during both the takeoff and landing phases with the dynamics governed by momentum theory. [29] The role of the vertical flight dynamics system is to determine the total rotor thrust and rotor power required to achieve the prescribed rate of climb. For this analysis, the total thrust required to attain a given climb rate is defined by Eq. 4 where induced velocity in hover $\left(v_{h}\right)$ is computed by Eq. 5. This thrust is then passed to the propulsion model along with the rate of climb to compute the necessary shaft input power.

$$
\begin{gathered}
T=2 \rho A_{\text {rotor }}\left(\dot{h}+V_{h}\right) V_{h} \\
V_{h}=\sqrt{\frac{T_{\text {rotor }}}{2 \rho A_{\text {rotor }}}}
\end{gathered}
$$

\section{Forward Flight}

Forward flight is modeled using a steady flight assumption and energy state approximation (Equations 6 and 7 ) originally posed by Bryson et al. whereby the flight path angle is assumed to be nearly zero. [30]

$$
\begin{gathered}
T \alpha+L(\alpha)=m g \\
\dot{E}=\frac{V_{\infty}(T-D)}{m g}
\end{gathered}
$$

In this equation, the total thrust $(T)$ of the aircraft at each point in time in forward-flight is imposed as a control variable. The aircraft lift $(L)$ is provided by the wing aerodynamics model and is a function of the angle-of-attack $(\alpha)$ of the vehicle. Finally, the vehicle weight is computed by summing of the masses of the constituent subsystems and accounting for the fuel mass which has been consumed by the propulsion system up until that point in the flight. As the relationship in Eq. 6 is implicit, a Newton solver is used to find the value of $\alpha$ which balances the equation.

\section{Turbo-Electric Tiltwing Optimization Demonstration Problem}

To evaluate the proposed multidisciplinary design environment and demonstrate the importance of tightly coupling the selected disciplines, the developed capability was applied in the study of the tiltwing vehicle described in the Introduction. The design specifications for this vehicle called for it to carry 15 passengers $(3000 \mathrm{lbm})$ on eight 50 $\mathrm{nm}$ flights before needing refueled. Additionally, the vehicle needs to be able to operate at high altitudes (takeoff and landing at $5000 \mathrm{ft}$ ) on hot days (ISA $+36^{\circ} \mathrm{R}$ ). The tiltwing vehicle was assumed to have a turboelectric propulsion system with a battery backup capable of providing 2 minutes of hover power for an emergency landing.

For the initial demonstration studies presented in this section, several simplifying assumptions were made for designing and analyzing this concept. First, the design trajectory was modified to evaluate a single $400 \mathrm{~nm}$ flight rather that the eight shorter flights. This approach was selected as it captures most of the overall mission performance requirements but eliminates the complexity of multiple takeoff and landing phases being modeled in the the trajectory. For this trajectory, the takeoff and landing were assumed to occur at $5000 \mathrm{ft}$ with the maximum allowable altitude at any point in the flight capped at 10,000 ft. Additionally, the gas turbine was assumed fixed with the power and fuel flow rates being scaled based on requirements from the rest of the propulsion system. These simplifying assumptions were made for this initial evaluation and demonstration of the multidisciplinary environment with the intention of removing these assumptions in future iterations of this research. 
Given these simplifying assumptions, a formal statement for the optimization problems evaluated in this initial demonstration of the modeling environment is stated in Table 2 Overall, the objective of the design studies was to modify the disciplinary designs and operating characteristics of the vehicle to minimize the fuel required for the assumed flight. This objective is stated in the top portion of the table. Below this section, the table lists the design variables considered for the tiltwing vehicle. This section is subdivided into three smaller sections to identify the mapping of the design variables to the disciplines under consideration: propulsion system design, wing aerostructural design, and trajectory analysis. Lastly, the bottom of the table lists the constraints placed on the design and trajectory along with the discipline to which they apply. For many of the design variables and constraints, a single scalar value was not sufficient to define available design space. Therefore, the size column in Table 2 specifies the length of the vector which was considered for each design variable and constraint. For example, the propeller blade chord design variable consisted of 6 unique values along the span of the blade which could be independently controlled by the optimizer. Similarly, for the altitude design variable in the trajectory 69 individual altitude variables were exposed to the optimizer along the trajectory in order for it to determine the best possible flight path.

Table 2 Tiltwing Optimization Problem Formulation.

\begin{tabular}{rlll}
\hline & Variable/Function & Size & Discipline \\
\hline minimize & Initial fuel mass & & \\
& Propeller blade chord & 6 & \\
& Propeller blade twist & 6 & Propulsion \\
& Generator Speed & 18 & \\
& Motor speed & 18 & \\
\cline { 2 - 4 } & Wing span & 1 & \\
& Wing sweep & 1 & \\
& Wing twist distribution & 3 & Wing aerostructural \\
& Wing skin thickness & 3 & \\
& Wing spar thickness & 3 & \\
\cline { 2 - 4 } & Altitude & 69 & \\
& Range & 37 & \\
& Velocity & 37 & \\
& Fuel mass & 69 & Trajectory \\
& Thrust (forward flight) & 10 & \\
& Acceleration (forward flight) & 4 & \\
& Blade pitch (forward flight) & 6 & \\
& Phase time boundaries & 5 & \\
\hline subject to & Fuel burn residual & 1 & Aircraft mass \\
& Stress constraints & 3 & Wing aerostructural \\
& Pseudospectral constraints & 210 & Trajectory \\
\hline
\end{tabular}

Using this general problem statement, four different optimizations were run to demonstrate the impact of coupling the various disciplinary analysis together on the tiltwing vehicle design. In each of these optimizations, the flight dynamics were modeled and the optimal trajectory was computed. However, for each optimization, different combinations of the disciplinary models were included. For disciplines that were not included, simplified low-fidelity models were inserted with fixed inputs extracted from the initial design produced by the traditional process. The first optimization problem used these simplified models for all of the disciplines and is referred to as the baseline configuration. In this baseline study, only the trajectory design variables were included in the optimization. Next, two optimizations were conducted which explored the design space formed by focusing on a single discipline in isolation to demonstrate the impact of a sequential, loosely coupled process. The first of these studies included the detailed aerostructural design of the wing along with the trajectory and therefore only considered the wing and trajectory design variables. Meanwhile, 
Table 3 Aircraft Sizing Optimization Results.

\begin{tabular}{lcccc}
\hline Parameter & Baseline & Wing Only & Propulsion Only & Fully Coupled \\
\hline Maximum takeoff weight, lbm & 13761 & 13621 & 12456 & 12588 \\
Empty weight, lbm & 9055 & 9029 & 7965 & 8148 \\
Fuel weight, lbm & 1705 & 1592 & 1490 & 1440 \\
Wing weight, lbm & 820 & 1658 & 820 & 1418 \\
Main structure weight, lbm & 1928 & 1907 & 1801 & 1807 \\
Rotor/propeller weight, lbm & 541 & 439 & 318 & 331 \\
Electric system weight, lbm & 3711 & 2970 & 2972 & 2536 \\
Gas turbine weight, lbm & 2055 & 2055 & 2055 & 2055 \\
Rotor/propeller diameter, ft & 11.73 & 15.34 & 11.73 & 14.57 \\
Wingspan, ft & 52.5 & 65.62 & 52.5 & 62.83 \\
\hline
\end{tabular}

the second evaluated only the design of the propulsion system, specifically the rotors/propellers and the electrical distribution system, in conjunction with the trajectory (using the propulsion and trajectory design variables). The fourth and final optimization study examined the full tiltwing design problem presented in Table 2 and considered all the design variables and constraints. It is important to note that the optimization results presented here are intended to show the value of the tightly-coupled multidisciplinary design environment, not identify the best available tiltwing design. Further refinement of the individual disciplinary tools and models along with evaluation by rotorcraft experts are ultimately needed to determine the validity of the designs produced.

The results for these four optimization studies are shown in Table 3 and Figures 10 to 12 . First, the major aircraft design and sizing parameters are provided in Table 3 . In this table, the first results column shows the baseline design produced by the simplified models implemented for each discipline. Overall, this system has approximately the same takeoff gross weight and empty weight as the design produced with the traditional process, indicating that the simplified models are generally representative. It should be noted that the weight values are slightly lower than those reported in the initial RVLT study [3] as the simplified $400 \mathrm{~nm}$ trajectory considered here does not include the numerous high power (and thereby high fuel flow) vertical takeoff and landing segments. For the flight path shown in Fig. 10, the baseline configuration (like each of the other studies) climbs several hundred feet in vertical flight then during forward flight climbs to the maximum allowable altitude for the duration of cruise before descending and landing again in a vertical decent. In this trajectory figure, the aircraft does not stay exactly at the maximum of 10,000 ft during the cruise portion of the flight. The subtle 'wiggles' observed here are an artifact of using a fairly coarse time discretization with the pseudospectral time integration scheme in Dymos. The coarse time scheme minimizes the computational cost when running the time integration, and the wiggles do not significantly impact the final integrated fuel burn values. Finally, Figures 11 and 12 show the total thrust and gas turbine power required throughout the flight.

The second optimization study considered examined only the aerostructural design of the wing along with the trajectory. The outputs from this study are labeled as 'Wing Only' and show a different vehicle design compared to the baseline study. Overall, the takeoff gross weight and the empty weight remain approximately the same as the baseline case as indicated by the hover and landing thrust in Fig. 11. Examining the design further, however, shows that the wingspan significantly increased (up to the maximum allowable) which also increased the wing weight. While increasing the wing weight seems undesirable, this change has a coupled impact on the simplified propulsion system model included in this study. In the propulsion system model, the rotor/propeller diameter was constrained by the wingspan and therefore the increase in span allowed the propeller diameter to also increase. The larger propellers which were selected are more efficient in hover and therefore require less power from the gas turbine as shown in Fig. 12. As the weight of the gas turbine, electrical system components and propellers are generally correlated with power, the reduction in required power lead to a lower the weight for these systems which offset the increase in wing weight.

The third optimization study examined the design of the propulsion system along with the trajectory in isolation from the rest of the detailed disciplinary design tools. The results from this optimization show takeoff gross weight and the empty weight were both reduce by over $1000 \mathrm{lbm}$ compared to the baseline design. This reduction came primarily from a lower weight estimate for the electrical system components, with a smaller weight reduction also achieved by refining 


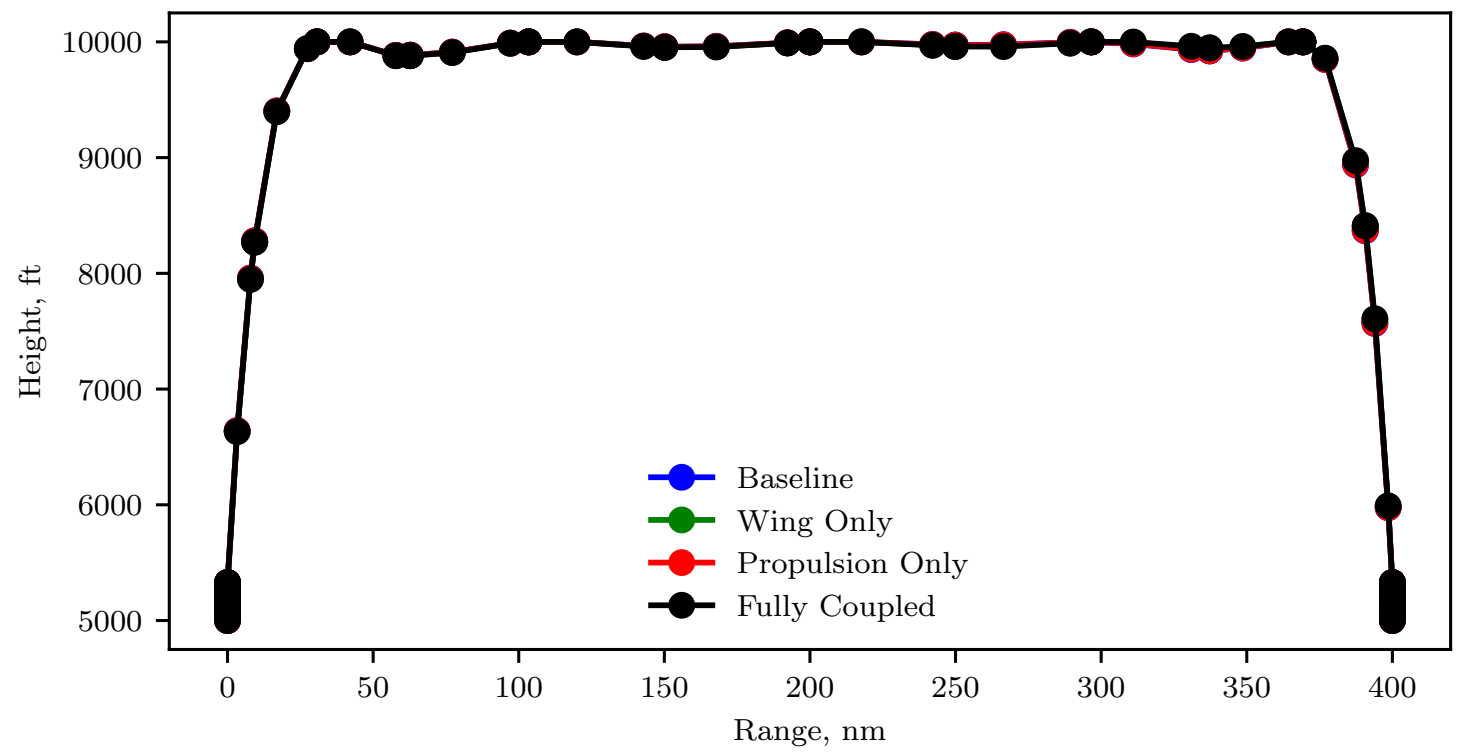

Fig. 10 Trajectory Optimization Results.

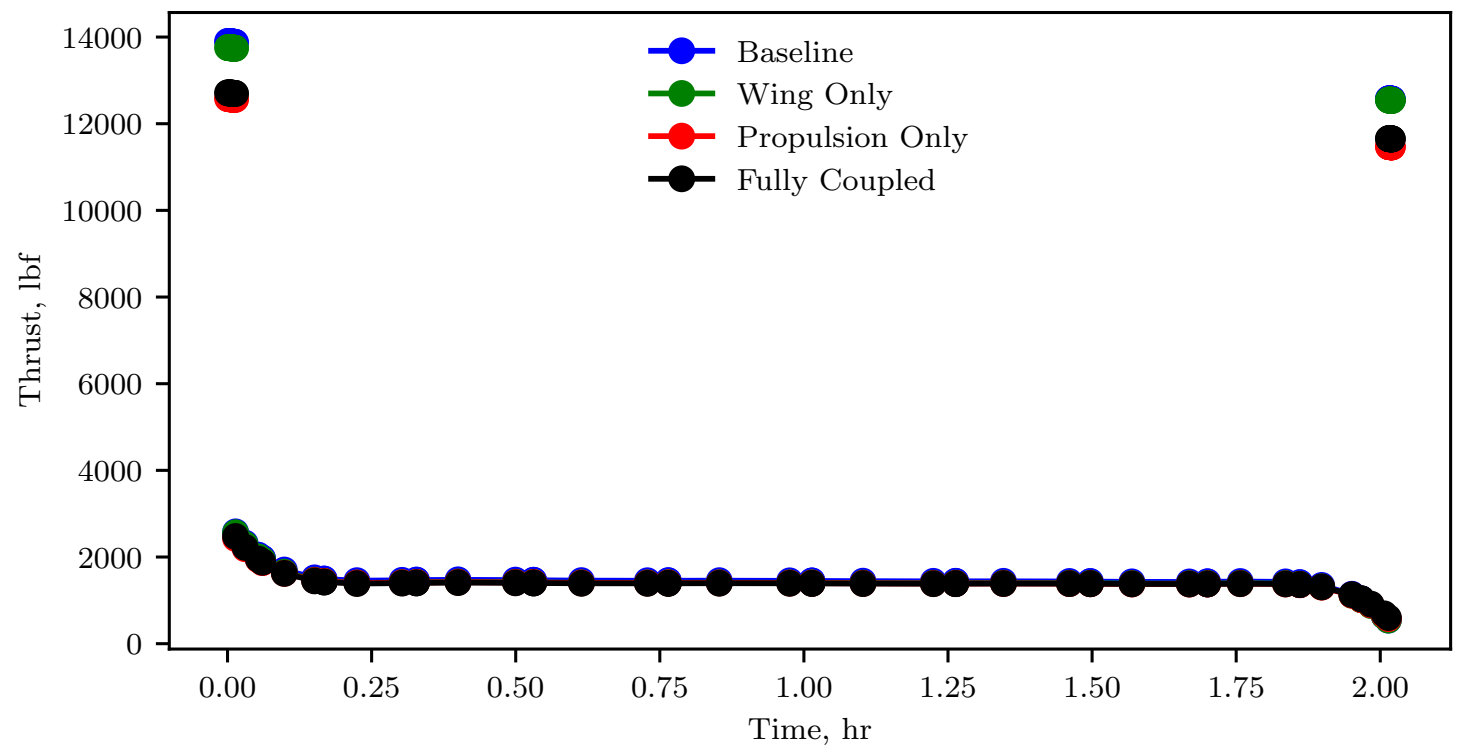

Fig. 11 Thrust Optimization Results.

the propeller design. Furthermore, the lower weights for these subsystems had a compounding effect on the vehicle size and thrust requirements which reduced the total fuel weight by $200 \mathrm{lbm}$. The reduced total thrust demand compared to the first two studies can be seen in the takeoff and landing portions of Fig. 11 . While the required thrust was lower for the propulsion system only optimization compared to the wing only and baseline studies, this reduction in thrust did not correspond to as significant a reduction in required power (Fig. 12). Here, the propeller diameter was limited by the fixed wingspan of the aircraft thereby preventing the propulsion system design from moving to larger more efficient rotors. The blade however was redesigned within this constraint with the optimal geometry shown in Fig.13. 


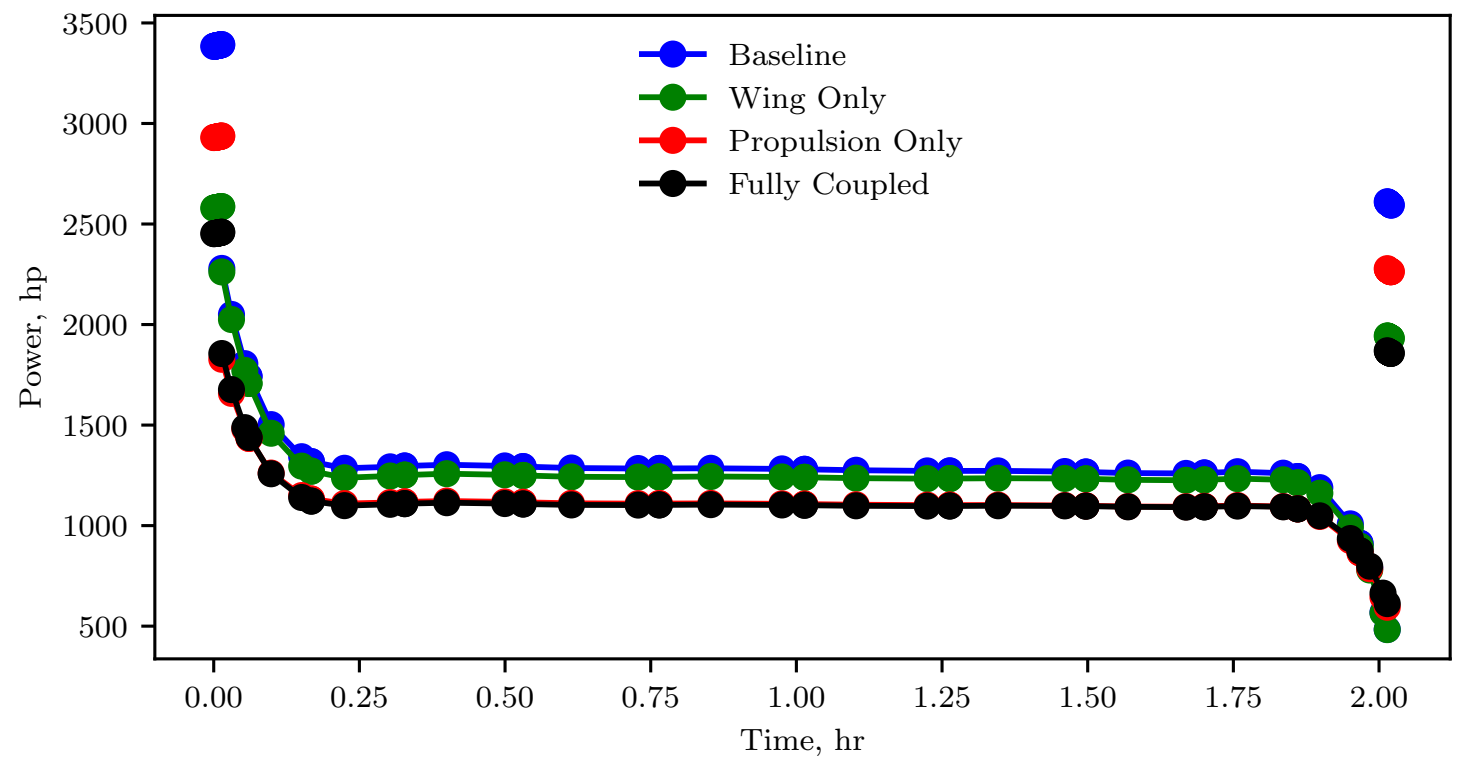

Fig. 12 Power Optimization Results.

Finally, the last optimization study considered the fully coupled design problem with detailed models for each of the disciplines. The results from this study show that the vehicle takeoff gross weight and empty weight are both lower than the baseline design, but are slightly higher than the propulsion only optimization. This increase is due to the coupling of the various disciplines which captures trade-offs and interactions between the propulsion system and wing designs. For the fully coupled problem, the best design was found to have a wingspan greater than the baseline design but less than the wing only optimized result. This design was still relatively heavy compared to the baseline, but by extending the span larger diameter propellers with improved performance were able to be designed. The larger diameter propeller design characteristics are shown in Fig. 13 Consequently, the power required (and therefore the total fuel burned) in both hover and forward flight for the fully coupled design was the lowest of any of the studies as shown in Fig. 12

In summary, the initial demonstration results presented in this section for several tiltwing vehicle studies show the importance of tightly coupling the disciplinary analysis. While the flight trajectories of individual studies did not vary significantly, the studies did reveal non-intuitive coupling between the wing and propulsion system design. Primarily, it was found that increasing the wingspan made the wing heavier but this change allowed the diameter of the propellers to increase leading to more efficient propeller designs. The improved propeller designs had the further benefit of reducing the power required from the gas turbine that would then need to be transmitted through the electrical distribution system. Given the weight of the electrical system components is correlated to the power they must handle, the reduction in power reduced the weight of these components and compounded to further reduce the weight of the vehicle. This lower vehicle weight and the improve performance both lead to a substantial reduction of the fuel mass required to complete the $400 \mathrm{~nm}$ mission.

\section{Conclusion and Future Work}

NASA's Revolutionary Vertical Lift Technology Project (RVLT) is exploring the design space for urban air mobility (UAM) vehicle concepts. These concepts cover a range of different designs but all explore the integration of emerging technology areas such as electrified propulsion systems. Exploration of the conceptual design space for these vehicles therefore presents challenges given new discipline analyses required and loose coupling traditionally considered between disciplines during these studies.

To address these challenges, this research developed a new tightly-coupled multidisciplinary design environment with the aim of better analyzing these unique vehicle concepts. This multidisciplinary environment was built on top of the OpenMDAO framework and applied gradient based optimization methods to explore the large design space for 

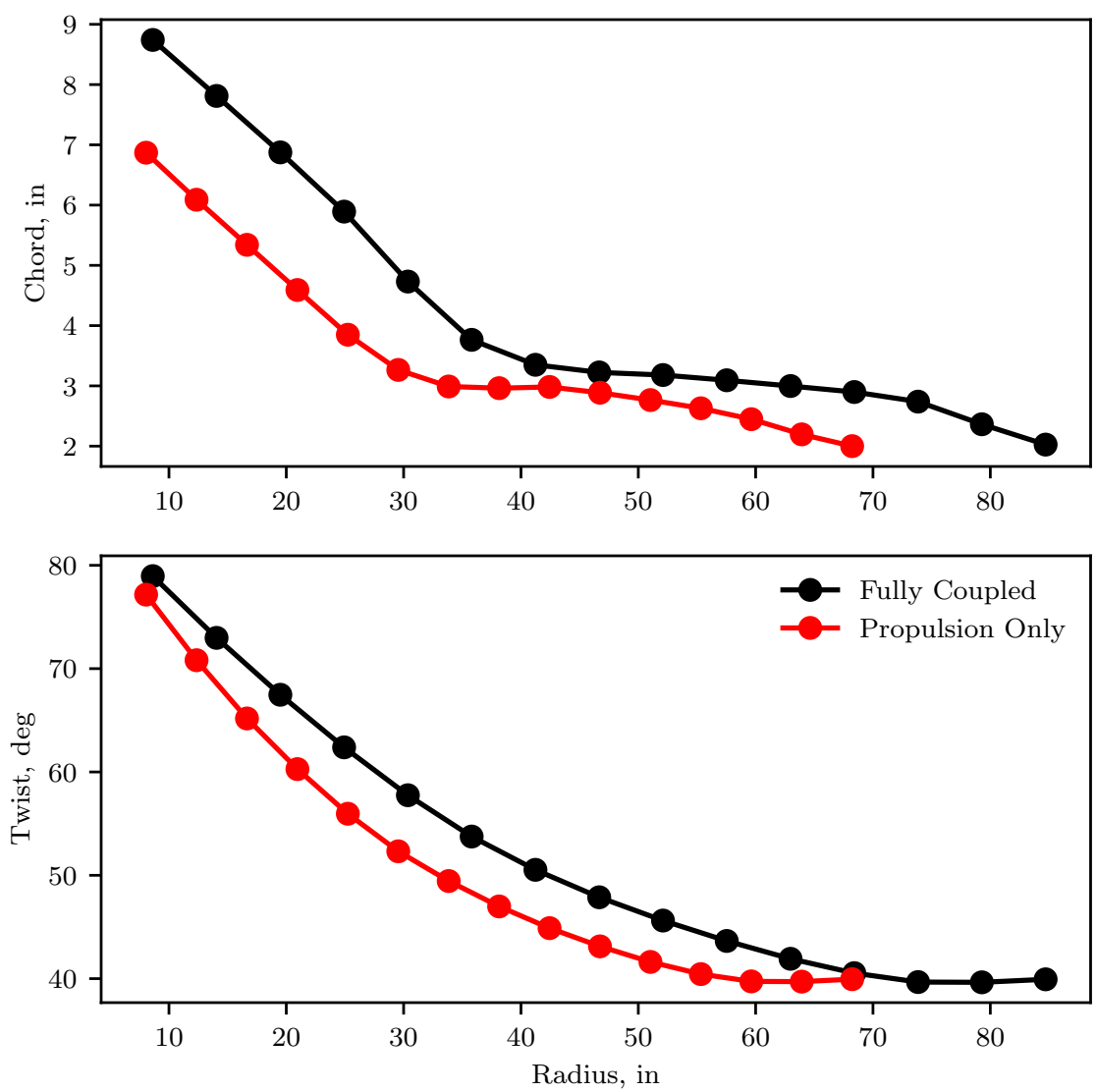

Fig. 13 Propeller Blade Optimization Results.

these vehicles. The use of gradient-based optimization is critical given the large design space and was facilitated by developing a new suite of disciplinary analysis tools which accurately and rapidly compute analytic derivatives needed in this process.

The newly developed multidisciplinary design and optimization environment was demonstrated in the analysis of a tiltwing aircraft capable of carrying 15 passengers on eight $50 \mathrm{~nm}$ flights. The focus of this demonstration was to investigate the importance of tightly integrating the various disciplines on the overall design, not on the actual designs produced by the analysis. Hence, a number of simplifying assumptions were made for the design problem presented, with the most significant being the modeling of a single $400 \mathrm{~nm}$ flight instead of eight short hops.

To show the value of tight coupling between the disciplines, a series of optimization studies were completed with different levels of modeling and coupling used between the disciplines. The baseline study, which used reduced order models for all disciplines, produced an aircraft that was comparable in design and performance to the initial design produced by the RVLT project. The next studies completed detailed designs of the wing and propulsion systems in isolation with each producing vehicle designs that showed improvements to the vehicle primarily in regards to that discipline. In each of these cases, the weight of the aircraft was reduced and the fuel required to complete the fixed mission also decreased. However, these studies did not take advantage of the coupled nature of the design problem. In the final, tightly-coupled optimization, further improvements were made to the overall design and performance characteristics with gains made by each of the disciplines. Specifically, the wing span of the vehicle was increased which added weight to the aircraft but allowed for a corresponding increase in propeller diameter leading to better efficiency 
and overall lower power requirements. These results demonstrated the value of the tightly-coupled multidisciplinary design and optimization environment for evaluating these types of vehicles.

Overall, the research described in this paper presented an initial attempt to tightly couple several of the major aircraft design disciplines within a multidisciplinary environment. While benefits were shown for tightly coupling these disciplines, it is recognized that there are several areas that need to be improved in future work. First, for several of the disciplines considered in this research relatively simple physics models were implemented which do not accurately capture the performance characteristics of the individual subsystems. This is particularly true for the blade element momentum theory representation of the propellers that evaluated them in isolation of the other propellers and wing. These disciplinary models therefore need to be upgrade to higher-fidelity physics with fewer significant assumptions in order provide confidence in the results produced by this method. Second, there are additional discipline areas that were not included in this study that could have significant impact on the overall design. One of these disciplines is a thermal management system which is likely required to dissipate the waste heat generated by the electrical system components. At this time, it is unclear if passive cooling techniques will be sufficient for these components or if active cooling systems will be required. Regardless of the form of the thermal management system, the inclusion of this system in the model would capture important coupling with the electrical system design and vehicle aerodynamics. Furthermore, the thermal system design is highly dependent on the transient operation of the vehicle which may have a coupled effect on the optimal flight path. In addition to the thermal discipline, the operation of the UAM concepts over densely populated city environments is likely to place additional requirements on reducing the noise produced by these vehicles. Therefore, inclusion of an acoustic disciplinary analysis, which will have coupling to the aircraft weight and propeller design, will be vital for developing a vehicle design and trajectory that enable operation in this environment.

\section{Acknowledgments}

The work presented in this paper is supported by NASA's Transformative Tools and Technologies (TTT) Project. The authors would like to thank Christopher Silva and NASA's Revolutionary Vertical Lift Technology (RVLT) project for their support in providing preliminary design characteristics for the tiltwing UAM concept vehicle. The authors would also like to thank Shamsheer Chauhan for his insight into wing design and modeling.

\section{References}

[1] Sillery, B., "Looking back at Henry Ford's Flivver," Popular Science, 2001. https://www.popsci.com/militaryaviation-space/article/2001-12/looking-back-henry-fords-flivver, Accessed: 2018-06-08.

[2] Moore, M. D., "Personal Air Vehicles: A Rural/Regional and Intra-Urban On-Demand Transportation System,” July 2003. doi:10.2514/6.2003-2646, AIAA paper 2003-2646.

[3] Johnson, W., Silva, C., and Solis, E., "Concept Vehicles for VTOL Air Taxi Operations," AHS International Technical Meeting on Aeromechanics Design for Transformative Vertical Flight, San Francisco, CA, 2018.

[4] Silva, C., Johnson, W., Antcliff, K. R., and Patterson, M. D., "VTOL Urban Air Mobility Concept Vehicles for Technology Development," 2018 Aviation Technology, Integration, and Operations Conference, 2018. doi:10.2514/6.2018-3847.

[5] Lyu, Z., Xu, Z., and Martins, J. R. R. A., "Benchmarking Optimization Algorithms for Wing Aerodynamic Design Optimization," 8th International Conference on Computational Fluid Dynamics (ICCFD8), Chengdu, China, 2014. ICCFD8 2014-0203.

[6] Hendricks, E. S., “A Multi-Level Multi-Design Point Approach for Gas Turbine Cycle and Turbine Conceptual Design,” PhD dissertation, Georgia Institute of Technology, School of Aerospace Engineering, 2017.

[7] Martins, J. R. R. A., Sturdza, P., and Alonso, J. J., “The Complex-Step Derivative Approximation,” ACM Transactions on Mathematical Software, Vol. 29, No. 3, 2003, p. 245-262. doi:10.1145/838250.838251.

[8] Gray, J. S., Hwang, J. T., Martins, J. R. R. A., Moore, K. T., and Naylor, B. A., "OpenMDAO: An Open-Source Framework for Multidisciplinary Design, Analysis, and Optimization,” Structural and Multidisciplinary Optimization, Vol. 59, 2019, pp. 1075-1104. doi:10.1007/s00158-019-02211-z.

[9] Lambe, A. B., and Martins, J. R. R. A., "Extensions to the Design Structure Matrix for the Description of Multidisciplinary Design, Analysis, and Optimization Processes," Structural and Multidisciplinary Optimization, Vol. 46, 2012 , pp. $273-284$. doi:10.1007/s00158-012-0763-y. 
[10] Claus, R. W., Evans, A., Lylte, J., and Nichols, L., "Numerical Propulsion System Simulation," Computing Systems in Engineering, Vol. 2, No. 4, 1991, pp. 357-364. doi:10.1016/0956-0521(91)90003-N.

[11] Johnson, W., "Technology Drivers in the Development of CAMRAD II," American Helicopter Society Aeromechanics Specialists Conference, San Francisco, California, 1994.

[12] Johnson, W., "Rotorcraft Aeromechanics Applications of a Comprehensive Analysis," HeliJapan 1998: AHS International Meeting on Advanced Rotorcraft Technology and Disaster Relief, Gifu, Japan, 1998.

[13] Johnson, W., "Rotorcraft Aerodynamics Models for a Comprehensive Analysis," American Helicopter Society 54th Annual Forum, Washington, D.C., 1998.

[14] Johnson, W., "NASA Design and Analysis of Rotorcraft Theory Manual,", 2012. https://rotorcraft.arc.nasa.gov/ Publications/files/NDARCTheory_v1_6_938.pdf

[15] Chittick, I. R., and Martins, J. R. R. A., "An Asymmetric Suboptimization Approach to Aerostructural Optimization," Optimization and Engineering, Vol. 10, No. 1, 2009, p. 133-152. doi:10.1007/s11081-008-9046-2.

[16] Falck, R. D., Chin, J. C., Schnulo, S. L., Burt, J. M., and Gray, J. S., "Trajectory Optimization of Electric Aircraft Subject to Subsystem Thermal Constraints," 18th AIAA/ISSMO Multidisciplinary Analysis and Optimization Conference, Denver, CO, 2017.

[17] Schnulo, S. L., Jeff Chin, R. D. F., Gray, J. S., Papathakis, K. V., Clarke, S. C., Reid, N., and Borer, N. K., "Development of a Multi-Segment Mission Planning Tool for SCEPTOR X-57," 2018 Multidisciplinary Analysis and Optimization Conference, AIAA, Atlanta, GA, 2018. doi:10.2514/6.2018-3738.

[18] Chapman, J. W., "Multi-point Design and Optimization of a Turboshaft Engine for Tiltwing turboelectric VTOL Air Taxi," Jan. 2019. doi:10.2514/6.2019-1948, AIAA paper 2019-1948.

[19] Falck, R. D., and Gray, J. S., "Optimal Control within the Context of Multidisciplinary Design, Analysis, and Optimization," AIAA Scitech 2019 Forum, AIAA, San Diego, CA, 2019. doi:10.2514/6.2019-0976.

[20] Andrew Ning, S., "A Simple Solution Method for the Blade Element Momentum Equations with Guaranteed Convergence," Wind Energy, 2013, p. n/a-n/a. doi:10.1002/we.1636.

[21] Hwang, J. T., and Ning, A., "Large-Scale Multidisciplinary Optimization of an Electric Aircraft for On-Demand Mobility," 2018 AIAA/ASCE/AHS/ASC Structures, Structural Dynamics, and Materials Conference, American Institute of Aeronautics and Astronautics, 2018. doi:10.2514/6.2018-1384.

[22] Ingraham, D., Gray, J. S., and Lopes, L. V., "Gradient-Based Propeller Optimization with Acoustic Constraints,” AIAA Scitech 2019 Forum, American Institute of Aeronautics and Astronautics, 2019. doi:10.2514/6.2019-1219.

[23] Hendricks, E. S., Chapman, J. W., and Aretskin-Hariton, E. D., "Load Flow Analysis with Analytic Derivatives for Electric Aircraft Design Optimization,” Jan. 2019. doi:10.2514/6.2019-1220, AIAA paper 2019-1220.

[24] Gray, J., Chin, J., Hearn, T., Hendricks, E., Lavelle, T., and Martins, J. R., "Chemical-Equilibrium Analysis with Adjoint Derivatives for Propulsion Cycle Analysis," Journal of Propulsion and Power, Vol. 33, No. 5, 2017, pp. 1041-1052. doi:10.2514/1.B36215.

[25] Hearn, T. A., Hendricks, E., Chin, J., and Gray, J. S., "Optimization of Turbine Engine Cycle Analysis with Analytic Derivatives," June 2016. doi:10.2514/6.2016-4297, AIAA paper 2016-4297.

[26] Jasa, J. P., Hwang, J. T., and Martins, J. R. R. A., “Open-Source Coupled Aerostructural Optimization using Python,” Structural and Multidisciplinary Optimization, Vol. 57, No. 4, 2018, pp. 1815-1827. doi:10.1007/s00158-018-1912-8.

[27] Raymer, D. P., Aircraft Design: A Conceptual Approach, $5^{\text {th }}$ ed., AIAA, 2012.

[28] Federal Aviation Administration, "Federal Aviation Regulations 25: Airworthiness Standards: Transport Category Airplanes," Washington, DC, Vol. 7, 2002.

[29] Leishman, G. J., Principles of Helicopter Aerodynamics with CD Extra, Cambridge university press, 2006.

[30] Bryson, A. E., Jr., Desai, M. N., and Hoffman, W. C., "Energy-State Approximation in Performance Optimization of Supersonic Aircraft," Journal of Aircraft, Vol. 6, No. 6, 1969, pp. 481-488. doi:10.2514/3.44093. 